\title{
The Geo/Geo/1+1 Queueing System with Negative Customers
}

\author{
Zhanyou Ma, ${ }^{1}$ Yalin Guo, ${ }^{1}$ Pengcheng Wang, ${ }^{1}$ and Yumei Hou ${ }^{2}$ \\ ${ }^{1}$ College of Science, Yanshan University, Qinhuangdao 066004, China \\ ${ }^{2}$ School of Economics \& Management, Yanshan University, Qinhuangdao 066004, China
}

Correspondence should be addressed to Yumei Hou; hym_1220@163.com

Received 11 September 2013; Accepted 28 October 2013

Academic Editor: Yuji Liu

Copyright (C) 2013 Zhanyou Ma et al. This is an open access article distributed under the Creative Commons Attribution License, which permits unrestricted use, distribution, and reproduction in any medium, provided the original work is properly cited.

\begin{abstract}
We study a Geo/Geo/1+1 queueing system with geometrical arrivals of both positive and negative customers in which killing strategies considered are removal of customers at the head $(\mathrm{RCH})$ and removal of customers at the end (RCE). Using quasi-birthdeath $(\mathrm{QBD})$ process and matrix-geometric solution method, we obtain the stationary distribution of the queue length, the average waiting time of a new arrival customer, and the probabilities of servers in busy or idle period, respectively. Finally, we analyze the effect of some related parameters on the system performance measures.
\end{abstract}

\section{Introduction}

In recent years, queueing system with negative arrivals (Gqueue) has become a research hotspot. Those studied models have been used to simulate computer communication system and the manufacturing system et al. The appropriate killing strategy must be determined in the queueing system with negative arrivals. Gelenbe et al. [1] investigated a single-sever G-queue system with the RCE and RCH killing strategies. Jain and Sigman [2] considered the effect of disaster on M/G/1 queueing system. Based on the model of the literature [2], Boucherie and Boxma [3] made a promotion and established a new killing strategy to allow a negative arrival for removing a random amount of positive customers which made the killing strategy tend to generalization.

Continuous time queueing systems with negative customers have been discussed extensively in the past years (see the literature [2-4]). In recent years, scholars pay more attention to the study of the discrete-time queueing systems with negative arrivals and obtain some significant results. Atencia and Moreno [5] adopted two different types of killing strategies ( $\mathrm{RCH}$ and disaster) to analyze the $\mathrm{Geo} / \mathrm{Geo} / 1$ queueing model with negative arrivals and obtained the stationary probability generating function of the queue length by solving balance equation in the system. Wang and Zhang [6] introduced negative customers into the retrial queue and deduced the stochastic decomposition law on discrete-time retrial G-queue. Jia and Chen [7] analyzed a Geom/Geom/1 queue with negative customers and single working vacation and derived the probabilities of the server in idle and busy period. Su and $\mathrm{Ma}$ [8] dealt with a Geom/Geom/1 Bernoulli feedback queue with single vacation, and the stationary distribution for the number of customers in the system and the stochastic decomposition were obtained. Chae et al. [9] extended the Geo/Geo/1 queue, with negative and positive customers to a GI/Geo/1 queue, and presented the stationary distribution of queue length and the sojourn time distributions under the RCH and RCE killing strategies. Wang et al. [10] studied a discrete-time on-off source queueing system with negative customers. By using Markovian processes approach technique, closed-form expressions of some performance measures in the system were derived, such as the stationary probability generating functions of the queue length, unfinished work, and the sojourn time distribution. Then, Wang et al. [11] investigated the $\mathrm{MMBP} / \mathrm{Geo} / 1$ queue with correlated positive and negative customer arrivals; then the average buffer content and the stationary probabilities were obtained.

For the single server queueing system with a vacation policy, if there are many customers in the system, they can be served until the vacation is completed. In this case, the waiting time of customers and the queue length in 


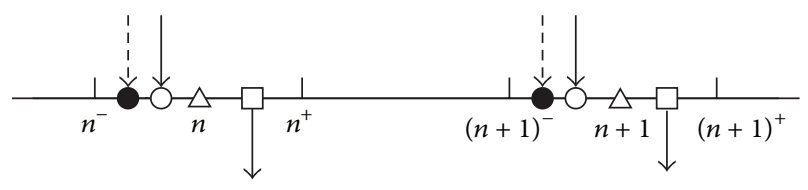

Arrival epoch of negative customers

$\triangle$ Beginning epoch of service

Arrival epoch of positive customers

Figure 1: The timetable of a late arrival system with delayed entrance.

the system can be increased. In order to solve these problems, a spare server will be started and serve customers when the number of customers reaches a certain number. This is the study purpose of the $\mathrm{Geo} / \mathrm{Geo} / 1+1$ queueing system; it can improve system efficiency by decreasing the waiting time of customers and the queue length. Such queue model can be found in many situations. For instant, a bank would supply a server when there are few customers to be served. If the number of customers increases to a fixed level, another server starts serving customers. Based on the above reason, we consider a $\mathrm{Geo} / \mathrm{Geo} / 1+1$ queue with negative customers in this paper, namely, adding a server based on the Geo/Geo/1 queueing system [12]. The two servers work at different rates. Negative customers are introduced into the system applying the RCH and RCE killing strategies to research it. By using QBD process and matrix-geometric solution, the stationary distributions and the average lengths of these two queues, the waiting time of a positive customer, and the probabilities of servers in busy period or idle period are derived.

The rest of this paper is organized as follows. In Section 2, we describe the queueing model and assume the system parameters and a service policy. In Section 3, we derive the transition probability matrix of the state in the queueing system with the $\mathrm{RCH}$ killing strategy and obtain the stationary probability distribution by using matrix geometric solution method. In Section 4, we study the similar analysis of the queueing system with the RCE killing strategy and obtain the corresponding theorem. In Section 5, we obtain some performance measures of the system, for example, the average queue length, the average waiting time, the state probabilities, and so on. Finally, by numerical examples, we analyze the effect of the parameters on the average queue length and the average waiting time of customer in Section 6.

\section{Model Description}

(1) We assume that interarrival times of positive and negative customers follow the geometrically distributed with probabilities $p(0<p<1)$ and $q(0<q<1)$. That is,

$$
\begin{array}{ll}
P\left(T_{1}=j\right)=p \bar{p}^{j-1}, & j=1,2, \ldots, \\
P\left(T_{2}=j\right)=q \bar{q}^{j-1}, & j=1,2, \ldots,
\end{array}
$$

where $\bar{p}=1-p, \bar{q}=1-q$.
(2) There are two servers in the system. When a new customer is coming, it gets into the limited queue II firstly. If the number of waiting customers in this queue reaches $K$, the new customer gets into the unlimited queue I. Service times for these two servers follow the geometrically distributed with probabilities $\mu_{2}\left(0<\mu_{2}<1\right)$ and $\mu_{1}\left(0<\mu_{1}<1\right)$. That is,

$$
\begin{array}{ll}
P\left(S_{1}=j\right)=\mu_{1} \bar{\mu}_{1}^{j-1}, & j=1,2, \ldots, \\
P\left(S_{2}=j\right)=\mu_{2} \bar{\mu}_{2}^{j-1}, & j=1,2, \ldots,
\end{array}
$$

where $\bar{\mu}_{1}=1-\mu_{1}, \bar{\mu}_{2}=1-\mu_{2}$.

(3) We consider a late arrival system with delayed entrance (see Figure 1): the arrivals of positive and negative customers occur in $\left(n^{-}, n\right)$, the ending of services occur in $\left(n, n^{+}\right)$, and the beginning of service occur at the epoch $n$.

(4) Interarrival times of positive and negative customers and service times for servers are assumed to be mutually independent. We assume that customers are served at least one time slot, and the service order is first-come first-served (FCFS) discipline.

\section{Steady State Analysis with the RCH Killing Strategy}

We analyze the Geo/Geo/1+1 queueing system with the $\mathrm{RCH}$ killing strategy in which a new negative arrival removes the positive customer being served at the head of the queue, and it will disappear when the queue is empty. The state transition probability matrix is derived by constituting a twodimensional Markov chain (MC). With matrix-geometric solution method, we obtain the matrix-geometric solution of the stationary distribution of the queue length in this system.

3.1. The State Transition Probability Matrix. This system can be described by a two-dimensional Markov chain $\left\{\left(L_{1}\left(n^{+}\right)\right.\right.$, $\left.\left.L_{2}\left(n^{+}\right)\right), n=1,2, \ldots\right\}$, where $L_{1}\left(n^{+}\right)$denotes the number of customers in the unlimited queue I and $L_{2}\left(n^{+}\right)$denotes the number of customers in the limited queue II. The state space is $\Omega=\{(i, j), i \geq 0,0 \leq j \leq K+1\}$.

By using the lexicographical order for the states, the transition probability matrix $\mathbf{P}$ can be written as the following matrix-block form:

$$
\mathbf{P}=\left[\begin{array}{ccccc}
\mathbf{A}_{00} & \mathbf{A}_{01} & & & \\
\mathbf{A}_{2} & \mathbf{A}_{1} & \mathbf{A}_{0} & & \\
& \mathbf{A}_{2} & \mathbf{A}_{1} & \mathbf{A}_{0} & \\
& & \ddots & \ddots & \ddots
\end{array}\right],
$$

where $\mathbf{A}_{00}, \mathbf{A}_{01}$, and $\mathbf{A}_{i}(i=0,1,2)$ are square matrices with $K+2$ order: 


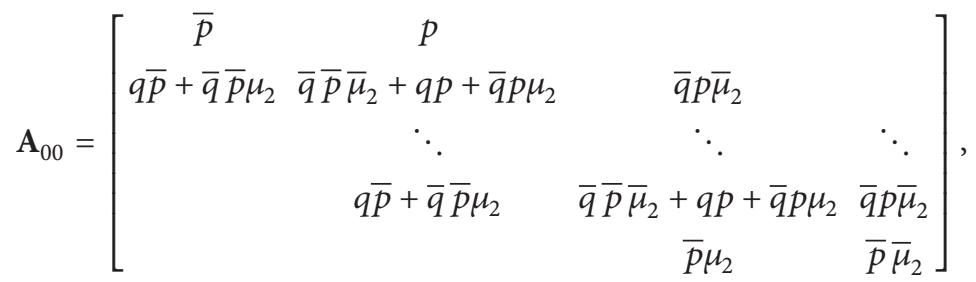

$$
\begin{aligned}
& \mathbf{A}_{01}=\left[\begin{array}{ccccc}
0 & \cdots & 0 & 0 & 0 \\
\vdots & & \vdots & \vdots & \vdots \\
0 & \cdots & 0 & 0 & 0 \\
0 & \cdots & 0 & p \mu_{2} & p \bar{\mu}_{2}
\end{array}\right], \quad \mathbf{A}_{0}=\left[\begin{array}{ccccc}
0 & \cdots & 0 & 0 & 0 \\
\vdots & & \vdots & \vdots & \vdots \\
0 & \cdots & 0 & 0 & 0 \\
0 & \cdots & 0 & \bar{q} p \mu_{2} \bar{\mu}_{1} & \bar{q} p \bar{\mu}_{2} \bar{\mu}_{1}
\end{array}\right] \text {, }
\end{aligned}
$$

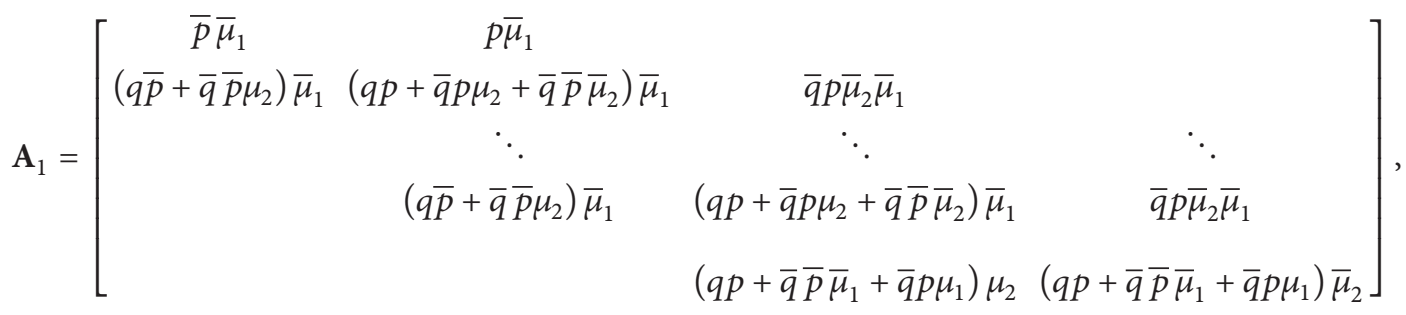

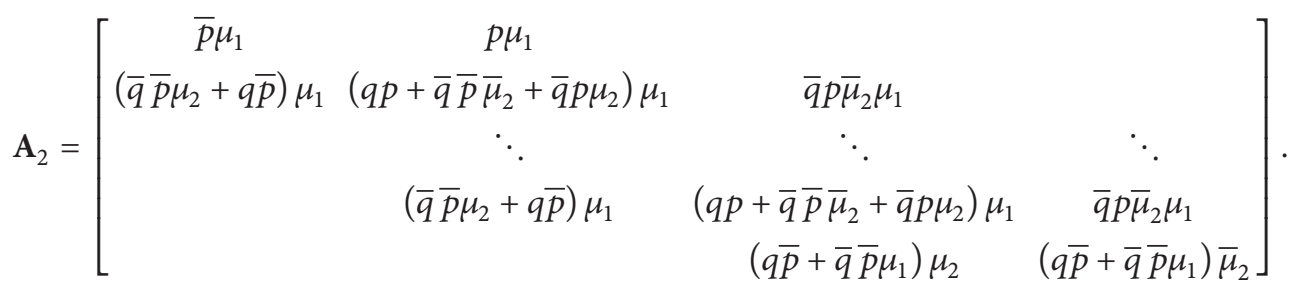

3.2. Stationary Probability Distribution. We denote $\left(L_{1}, L_{2}\right)$ as the stationary limit of $\left\{\left(L_{1}\left(n^{+}\right), L_{2}\left(n^{+}\right)\right), n=1,2, \ldots\right\}$ if the MC is ergodic. According to the structure of matrix $\mathbf{P}$, the stationary distribution of the queue length can be changed to the following form:

$$
\Pi=\left(\pi_{0}, \pi_{1}, \pi_{2}, \ldots\right),
$$

where

$$
\begin{gathered}
\pi_{i}=\left(\pi_{i, 0}, \pi_{i, 1}, \ldots, \pi_{i, K+1}\right), \quad i \geq 0, \\
\pi_{i, j}=P\left(L_{1}=i, L_{2}=j\right) \\
=\lim _{n \rightarrow \infty} P\left(L_{1}\left(n^{+}\right)=i, L_{2}\left(n^{+}\right)=j\right), \\
\quad i \geq 0,0 \leq j \leq K+1 .
\end{gathered}
$$

Theorem 1. The two-dimensional $M C\left\{\left(L_{1}\left(n^{+}\right), L_{2}\left(n^{+}\right)\right), n=\right.$ $1,2, \ldots\}$ is positive recurrent if and only if the spectral radius $\mathrm{SP}(\mathbf{R})$ of the minimal nonnegative solution $\mathbf{R}$ of the matrix equation

$$
\mathbf{R}^{2} \mathbf{A}_{2}+\mathbf{R} \mathbf{A}_{1}+\mathbf{A}_{0}=\mathbf{R}
$$

is less than 1. Then,

$$
B[\mathbf{R}]=\left[\begin{array}{cc}
\mathbf{A}_{00} & \mathbf{A}_{01} \\
\mathbf{A}_{2} & \mathbf{A}_{1}+\mathbf{R A}_{2}
\end{array}\right]
$$

is a stochastic matrix with $2(K+2)$ order and exists a left invariant vector $\left(\pi_{0}, \pi_{1}\right)$. When $M C$ is positive recurrent, the stationary distribution of the queue length is given by

$$
\begin{gathered}
\left(\boldsymbol{\pi}_{0}, \boldsymbol{\pi}_{1}\right) B[\mathbf{R}]=\left(\boldsymbol{\pi}_{0}, \boldsymbol{\pi}_{1}\right), \\
\boldsymbol{\pi}_{0} \mathbf{e}+\boldsymbol{\pi}_{1}(\mathbf{I}-\mathbf{R})^{-1} \mathbf{e}=1, \\
\boldsymbol{\pi}_{k}=\boldsymbol{\pi}_{1} \mathbf{R}^{k-1}, \quad k \geq 1,
\end{gathered}
$$

where $\mathbf{e}$ is a column vector of appropriate size with all components being 1 .

Proof. Since $\mathbf{P}$ is a stochastic matrix, we get $\left(\mathbf{A}_{00}+\mathbf{A}_{01}\right) \mathbf{e}=\mathbf{e}$. Due to $\mathrm{SP}(\mathbf{R})<1$, the matrix $\mathbf{I}-\mathbf{R}$ is invertible. We can find

$$
\begin{aligned}
\left(\mathbf{A}_{2}\right. & \left.+\mathbf{A}_{1}+\mathbf{R} \mathbf{A}_{2}\right) \mathbf{e} \\
& =\left[(\mathbf{I}+\mathbf{R}) \mathbf{A}_{2}+\mathbf{A}_{1}\right] \mathbf{e} \\
& =(\mathbf{I}-\mathbf{R})^{-1}\left[\mathbf{A}_{2}+\mathbf{A}_{1}-\left(\mathbf{R}^{2} \mathbf{A}_{2}+\mathbf{R} \mathbf{A}_{1}\right)\right] \mathbf{e} \\
& =\mathbf{e} .
\end{aligned}
$$


Expanding the balance equation of system states in this system, we have

$$
\begin{gathered}
\boldsymbol{\pi}_{0} \mathbf{A}_{00}+\pi_{1} \mathbf{A}_{2}=\pi_{0}, \\
\boldsymbol{\pi}_{0} \mathbf{A}_{01}+\pi_{1} \mathbf{A}_{1}+\pi_{2} \mathbf{A}_{2}=\pi_{1}, \\
\boldsymbol{\pi}_{k-1} \mathbf{A}_{0}+\pi_{k} \mathbf{A}_{1}+\pi_{k+1} \mathbf{A}_{2}=\pi_{k}, \quad k \geq 2 .
\end{gathered}
$$

Because $B[\mathbf{R}]$ exists a left invariant vector $\left(\boldsymbol{\pi}_{0}, \boldsymbol{\pi}_{1}\right)$, we can get

$$
\left(\boldsymbol{\pi}_{0}, \boldsymbol{\pi}_{1}\right) B[\mathbf{R}]=\left(\boldsymbol{\pi}_{0}, \boldsymbol{\pi}_{1}\right) .
$$

From (12), it is easy to find that the first equation and the second equation in (11) are valid obviously.

By substituting $\boldsymbol{\pi}_{k}=\pi_{1} \mathbf{R}^{k-1}, k \geq 1$ into the left-hand side of the third equation in (11), we can obtain

$$
\begin{aligned}
\boldsymbol{\pi}_{k-1} \mathbf{A}_{0} & +\boldsymbol{\pi}_{k} \mathbf{A}_{1}+\boldsymbol{\pi}_{k+1} \mathbf{A}_{2} \\
& =\boldsymbol{\pi}_{1} \mathbf{R}^{k-2}\left(\mathbf{A}_{0}+\mathbf{R} \mathbf{A}_{1}+\mathbf{R}^{2} \mathbf{A}_{2}\right)=\boldsymbol{\pi}_{k} .
\end{aligned}
$$

Using the normalization condition $\pi_{0} \mathbf{e}+\pi_{1} \mathbf{e}+\pi_{2} \mathbf{e}+\pi_{3} \mathbf{e}+$ $\cdots=1$ and substituting $\pi_{k}=\pi_{1} \mathbf{R}^{k-1}, k \geq 1$ into it, we have

$$
\pi_{0} \mathbf{e}+\pi_{1}\left(\mathbf{I}+\mathbf{R}+\mathbf{R}^{2}+\cdots\right) \mathbf{e}=1 .
$$

Because of $\mathrm{SP}(\mathbf{R})<1$, matrix power series $\mathbf{I}+\mathbf{R}+\mathbf{R}^{2}+\cdots$ converges to $(\mathbf{I}-\mathbf{R})^{-1}$. Equation (14) becomes

$$
\pi_{0} \mathbf{e}+\pi_{1}(\mathbf{I}-\mathbf{R})^{-1} \mathbf{e}=1
$$

\section{Steady State Analysis with the RCE Killing Strategy}

When we apply the RCE killing strategy to investigate this system, a negative customer immediately removes the positive customer at the end of the queue, and it will disappear if there is no customer in this queue. The stationary distribution of the queue length of the system can be derived by using the above method for $\mathrm{Geo} / \mathrm{Geo} / 1+1$ queueing system with the RCH killing strategy.

4.1. The State Transition Probability Matrix. We regard $L_{1}\left(n^{+}\right)$ and $L_{2}\left(n^{+}\right)$as the number of customers in the unlimited queue I and the number of customers in the limited queue II. It can be found that $\left\{\left(L_{1}\left(n^{+}\right), L_{2}\left(n^{+}\right)\right), n=1,2, \ldots\right\}$ is the Markov chain of the system whose state space is $\bar{\Omega}=$ $\{(i, j), i \geq 0,0 \leq j \leq K+1\}$.

Using the lexicographical order, the transition probability matrix can be described as

$$
\overline{\mathbf{P}}=\left[\begin{array}{ccccccc}
\mathbf{B}_{00} & \mathbf{B}_{01} & & & & \\
\mathbf{B}_{10} & \mathbf{B}_{11} & \mathbf{B}_{12} & & & \\
\mathbf{B}_{3} & \mathbf{B}_{2} & \mathbf{B}_{1} & \mathbf{B}_{0} & & \\
& \mathbf{B}_{3} & \mathbf{B}_{2} & \mathbf{B}_{1} & \mathbf{B}_{0} & \\
& & \ddots & \ddots & \ddots & \ddots
\end{array}\right],
$$

where $\mathbf{B}_{0 j}(j=0,1), \mathbf{B}_{1 j}(j=0,1,2)$, and $\mathbf{B}_{i}(i=1,2,3,4)$ are the following square matrices with $K+2$ order:

$$
\begin{aligned}
& \mathbf{B}_{00}=\left[\begin{array}{ccccc}
\bar{p} & p & & & \\
\left(q+\bar{q} \mu_{2}\right) \bar{p} & q p+c \bar{q} & \bar{q} p \bar{\mu}_{2} & & \\
q \bar{p} \mu_{2} & q \bar{p} \bar{\mu}_{2}+a \mu_{2} & \bar{q} p \mu_{2}+a \bar{\mu}_{2} & \bar{q} p \bar{\mu}_{2} & \\
& \ddots & \ddots & \ddots & \ddots \\
& q \bar{p} \mu_{2} & q \bar{p} \bar{\mu}_{2}+a \mu_{2} & \bar{q} p \mu_{2}+a \bar{\mu}_{2} & \bar{q} p \bar{\mu}_{2} \\
& & & \bar{p} \mu_{2} & \bar{p} \bar{\mu}_{2}
\end{array}\right], \\
& \mathbf{B}_{01}=\left[\begin{array}{ccccc}
0 & \cdots & 0 & 0 & 0 \\
\vdots & & \vdots & \vdots & \vdots \\
0 & \cdots & 0 & 0 & 0 \\
0 & \cdots & 0 & p \mu_{2} & p \bar{\mu}_{2}
\end{array}\right], \quad \mathbf{B}_{0}=\left[\begin{array}{ccccc}
0 & \cdots & 0 & 0 & 0 \\
\vdots & & \vdots & \vdots & \vdots \\
0 & \cdots & 0 & 0 & 0 \\
0 & \cdots & 0 & \bar{q} p \mu_{2} \bar{\mu}_{1} & \bar{q} p \bar{\mu}_{2} \bar{\mu}_{1}
\end{array}\right], \\
& \mathbf{B}_{10}=\left[\begin{array}{ccccc}
\bar{p} \mu_{1} & p \mu_{1} & & & \\
\left(q+\bar{q} \mu_{2}\right) \bar{p} \mu_{1} & (q p+c \bar{q}) \mu_{1} & \bar{q} p \bar{\mu}_{2} \mu_{1} & & \\
q \bar{p} \mu_{2} \mu_{1} & \left(q \bar{p} \bar{\mu}_{2}+a \mu_{2}\right) \mu_{1} & \left(\bar{q} p \mu_{2}+a \bar{\mu}_{2}\right) \mu_{1} & \bar{q} p \bar{\mu}_{2} \mu_{1} & \\
& \ddots & \ddots & \ddots & \ddots \\
& q \bar{p} \mu_{2} \mu_{1} & \left(q \bar{p} \bar{\mu}_{2}+a \mu_{2}\right) \mu_{1} & \left(\bar{q} p \mu_{2}+a \bar{\mu}_{2}\right) \mu_{1} & \bar{q} p \bar{\mu}_{2} \mu_{1} \\
& & & \left(q+\bar{q} \mu_{1}\right) \bar{p} \mu_{2} & \left(q+\bar{q} \mu_{1}\right) \bar{p} \bar{\mu}_{2}
\end{array}\right]
\end{aligned}
$$




$$
\begin{aligned}
& \mathbf{B}_{11}=\left[\begin{array}{ccccc}
\bar{p} \bar{\mu}_{1} & p \bar{\mu}_{1} & & & \\
\left(q+\bar{q} \mu_{2}\right) \bar{p} \bar{\mu}_{1} & (q p+c \bar{q}) \bar{\mu}_{1} & \bar{q} p \bar{\mu}_{2} \bar{\mu}_{1} & & \\
q \bar{p} \mu_{2} \bar{\mu}_{1} & \left(q \bar{p} \bar{\mu}_{2}+a \mu_{2}\right) \bar{\mu}_{1} & \left(\bar{q} p \mu_{2}+a \bar{\mu}_{2}\right) \bar{\mu}_{1} & \bar{q} p \bar{\mu}_{2} \bar{\mu}_{1} & \\
& \ddots & \ddots & \ddots & \ddots \\
& q \bar{p} \mu_{2} \bar{\mu}_{1} & \left(q \bar{p} \bar{\mu}_{2}+a \mu_{2}\right) \bar{\mu}_{1} & \left(\bar{q} p \mu_{2}+a \bar{\mu}_{2}\right) \bar{\mu}_{1} & \bar{q} p \bar{\mu}_{2} \bar{\mu}_{1} \\
& & & (q p+b \bar{q}) \mu_{2} & (q p+b \bar{q}) \bar{\mu}_{2}
\end{array}\right] \\
& \mathbf{B}_{12}=\left[\begin{array}{ccccc}
0 & \cdots & 0 & 0 & 0 \\
\vdots & & \vdots & \vdots & \vdots \\
0 & \cdots & 0 & 0 & 0 \\
0 & \cdots & 0 & \bar{q} p \mu_{2} \bar{\mu}_{1} & \bar{q} p \bar{\mu}_{2} \bar{\mu}_{1}
\end{array}\right], \quad \mathbf{B}_{3}=\left[\begin{array}{ccccc}
0 & \cdots & 0 & 0 & 0 \\
\vdots & & \vdots & \vdots & \vdots \\
0 & \cdots & 0 & 0 & 0 \\
0 & \cdots & 0 & q \bar{p} \mu_{2} \mu_{1} & q \bar{p} \bar{\mu}_{2} \mu_{1}
\end{array}\right], \\
& \mathbf{B}_{2}=\left[\begin{array}{ccccc}
\bar{p} \mu_{1} & p \mu_{1} & & & \\
\left(q+\bar{q} \mu_{2}\right) \bar{p} \mu_{1} & (q p+c \bar{q}) \mu_{1} & \bar{q} p \bar{\mu}_{2} \mu_{1} & & \\
q \bar{p} \mu_{2} \mu_{1} & \left(q \bar{p} \bar{\mu}_{2}+a \mu_{2}\right) \mu_{1} & \left(\bar{q} p \mu_{2}+a \bar{\mu}_{2}\right) \mu_{1} & \bar{q} p \bar{\mu}_{2} \mu_{1} & \\
& \ddots & \ddots & \ddots & \ddots \\
& q \bar{p} \mu_{2} \mu_{1} & \left(q \bar{p} \bar{\mu}_{2}+a \mu_{2}\right) \mu_{1} & \left(\bar{q} p \mu_{2}+a \bar{\mu}_{2}\right) \mu_{1} & \bar{q} p \bar{\mu}_{2} \mu_{1} \\
& & & \left(q \bar{p} \bar{\mu}_{1}+a \mu_{1}\right) \mu_{2} & \left(q \bar{p} \bar{\mu}_{1}+a \mu_{1}\right) \bar{\mu}_{2}
\end{array}\right] \text {, } \\
& \mathbf{B}_{1}=\left[\begin{array}{ccccc}
\bar{p} \bar{\mu}_{1} & p \bar{\mu}_{1} & & & \\
\left(q+\bar{q} \mu_{2}\right) \bar{p} \bar{\mu}_{1} & (q p+c \bar{q}) \bar{\mu}_{1} & \bar{q} p \bar{\mu}_{2} \bar{\mu}_{1} & & \\
q \bar{p} \mu_{2} \bar{\mu}_{1} & \left(q \bar{p} \bar{\mu}_{2}+a \mu_{2}\right) \bar{\mu}_{1} & \left(\bar{q} p \mu_{2}+a \bar{\mu}_{2}\right) \bar{\mu}_{1} & \bar{q} p \bar{\mu}_{2} \bar{\mu}_{1} & \\
& \ddots & \ddots & \ddots & \ddots \\
& q \bar{p} \mu_{2} \bar{\mu}_{1} & \left(q \bar{p} \bar{\mu}_{2}+a \mu_{2}\right) \bar{\mu}_{1} & \left(\bar{q} p \mu_{2}+a \bar{\mu}_{2}\right) \bar{\mu}_{1} & \bar{q} p \bar{\mu}_{2} \bar{\mu}_{1} \\
& & & \left(\bar{q} p \mu_{1}+a \bar{\mu}_{1}\right) \mu_{2} & \left(\bar{q} p \mu_{1}+a \bar{\mu}_{1}\right) \bar{\mu}_{2}
\end{array}\right],
\end{aligned}
$$

where $a=\bar{q} \bar{p}+q p, b=\bar{p} \bar{\mu}_{1}+p \mu_{1}$, and $c=\bar{p} \bar{\mu}_{2}+$ $p \mu_{2}$.

4.2. Stationary Probability Distribution. Define $\Pi=\left(\boldsymbol{\pi}_{0}, \boldsymbol{\pi}_{1}\right.$, $\left.\pi_{2}, \ldots\right)$ as the stationary distribution of the queue length of the system. The results of the $\mathrm{Geo} / \mathrm{Geo} / 1+1$ queueing system with the RCE killing strategy can be obtained as follows.

Theorem 2. The two-dimensional MC $\left\{\left(L_{1}\left(n^{+}\right), L_{2}\left(n^{+}\right)\right), n=\right.$ $1,2, \ldots\}$ is ergodic if and only if the spectral radius $\operatorname{SP}(\overline{\mathbf{R}})$ is less than 1, where $\overline{\mathbf{R}}$ is the minimal nonnegative solution of the matrix equation

$$
\overline{\mathbf{R}}^{3} \mathbf{B}_{3}+\overline{\mathbf{R}}^{2} \mathbf{B}_{2}+\overline{\mathbf{R}} \mathbf{B}_{1}+\mathbf{B}_{0}=\overline{\mathbf{R}} .
$$

And the stochastic matrix with $3(K+2)$ order

$$
B[\overline{\mathbf{R}}]=\left[\begin{array}{ccc}
\mathbf{B}_{00} & \mathbf{B}_{01} & \\
\mathbf{B}_{10} & \mathbf{B}_{11} & \mathbf{B}_{12} \\
\mathbf{B}_{3} & \mathbf{B}_{2}+\overline{\mathbf{R}} \mathbf{B}_{3} & \mathbf{B}_{1}+\overline{\mathbf{R}} \mathbf{B}_{2}+\overline{\mathbf{R}}^{2} \mathbf{B}_{3}
\end{array}\right]
$$

exists a left invariant vector $\left(\boldsymbol{\pi}_{0}, \boldsymbol{\pi}_{1}, \boldsymbol{\pi}_{2}\right)$. When $M C$ is ergodic, the stationary distribution is given by

$$
\begin{gathered}
\boldsymbol{\pi}_{k}=\boldsymbol{\pi}_{2} \overline{\mathbf{R}}^{k-2}, \quad k \geq 2, \\
\left(\boldsymbol{\pi}_{0}, \boldsymbol{\pi}_{1}, \boldsymbol{\pi}_{2}\right) B[\overline{\mathbf{R}}]=\left(\boldsymbol{\pi}_{0}, \boldsymbol{\pi}_{1}, \boldsymbol{\pi}_{2}\right), \\
\boldsymbol{\pi}_{0} \mathbf{e}+\boldsymbol{\pi}_{1} \mathbf{e}+\boldsymbol{\pi}_{2}(\mathbf{I}-\overline{\mathbf{R}})^{-1} \mathbf{e}=1,
\end{gathered}
$$

where $\mathbf{e}$ is a column vector with $K+2$ order in which all elements are 1.

The proof of Theorem 2 is as similar as the proof of Theorem 1.

\section{Performance Measures of the System}

5.1. The Stationary Queue Length. Denote $P_{1 i}$ and $P_{2 j}$ as the limit distribution of the unlimited queue I and the limited queue II, respectively; then 


$$
\begin{aligned}
P_{1 i} & =P\left(L_{1}=i\right)=\lim _{n \rightarrow \infty} P\left(L_{1}\left(n^{+}\right)=i\right) \\
& =\pi_{i} \mathbf{e}=\sum_{j=0}^{K+1} \pi_{i, j}, \quad i \geq 0, \\
P_{2 j} & =P\left(L_{2}=j\right)=\lim _{n \rightarrow \infty} P\left(L_{2}\left(n^{+}\right)=j\right)=\sum_{i=0}^{\infty} \pi_{i, j}, \\
& 0 \leq j \leq K+1 .
\end{aligned}
$$

The average queue length of the unlimited queue I and the limited queue II are given by

$$
\begin{gathered}
E\left(L_{1}\right)=\sum_{i=0}^{\infty} i P\left(L_{1}=i\right)=\sum_{i=0}^{\infty} i \sum_{j=0}^{K+1} \pi_{i, j}, \\
E\left(L_{2}\right)=\sum_{j=0}^{K+1} j P\left(L_{2}=j\right)=\sum_{j=0}^{K+1} j \sum_{i=0}^{\infty} \pi_{i, j} .
\end{gathered}
$$

The average waiting queue lengths are given by

$$
\begin{gathered}
E\left(Q_{1}\right)=\sum_{i=1}^{\infty}(i-1) P\left(L_{1}=i\right)=\sum_{i=1}^{\infty}(i-1) \sum_{j=0}^{K+1} \pi_{i, j}, \\
E\left(Q_{2}\right)=\sum_{j=1}^{K+1}(j-1) P\left(L_{2}=j\right)=\sum_{j=1}^{K+1}(j-1) \sum_{i=0}^{\infty} \pi_{i, j} .
\end{gathered}
$$

5.2. The Average Waiting Time of a Positive Arrival. Since negative customers cannot be served, and their waiting time is zero, the waiting time of a customer is just the waiting time of positive customers. A new positive arrival gets into the unlimited queue I with probability $\sum_{i=0}^{\infty} \pi_{i, K+1}$ and gets into the limited queue II with probability $1-\sum_{i=0}^{\infty} \pi_{i, K+1}$. According to Little's formula, we have

$$
E(W)=\sum_{i=0}^{\infty} \pi_{i, K+1} \frac{E\left(Q_{1}\right)}{\mu_{1}}+\left(1-\sum_{i=0}^{\infty} \pi_{i, K+1}\right) \frac{E\left(Q_{2}\right)}{\mu_{2}} .
$$

5.3. State Probabilities of Servers. Two states can be shown for these two servers: they are busy state, and idle state, respectively. Let server I be the server in the unlimited queue I and server II the server in the limited queue II. We use subscripts $b$ and $i$ to denote the busy state and the idle state, and obtain the following conclusions.

(1) The probability that server I is busy and server II is idle is given by

$$
P_{b i}=\sum_{i=1}^{\infty} \pi_{i, 0}
$$

(2) The probability that server I is idle and server II is busy is given by

$$
P_{i b}=\sum_{j=1}^{K+1} \pi_{0, j}
$$

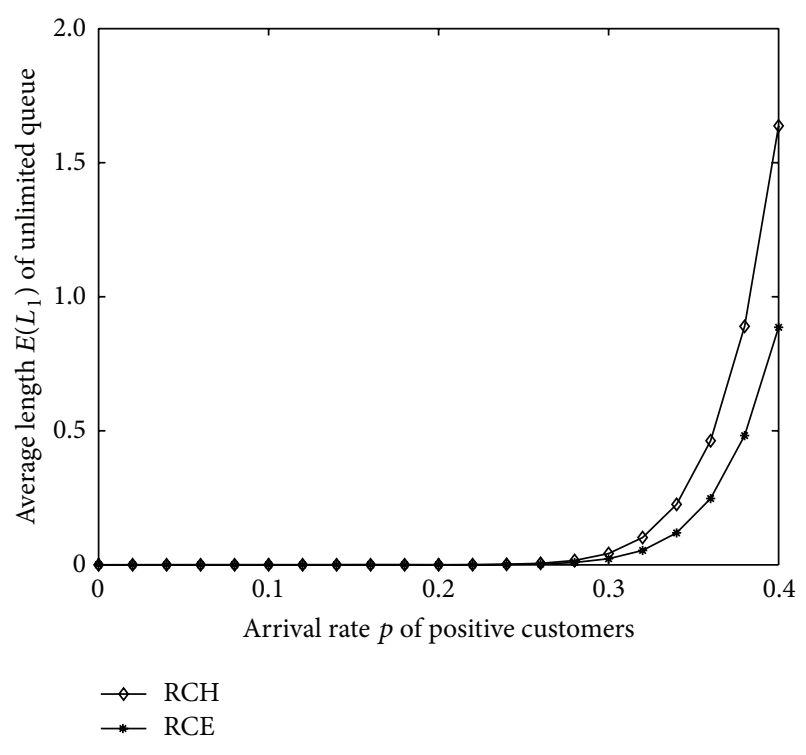

FIGURE 2: The effect of $p$ on $E\left(L_{1}\right)$.

(3) The probability that both of servers are in busy period is given by

$$
P_{b b}=\sum_{i=1}^{\infty} \sum_{j=1}^{K+1} \pi_{i, j}
$$

\section{Numerical Examples}

Through the previously mentioned analysis, we have got the expressions of the main performance measures with two kinds of strategies. By observing the following curve charts, we investigate the effect of parameters on the performance measures.

Figures 2 and 3 are drawn in the condition of $\mu_{1}=0.1$, $\mu_{2}=0.3, q=0.1$, and $K=10$. Figure 2 describes the impact of $p$ on the average length of unlimited queue. When $p$ changes from 0 to $0.2, E\left(L_{1}\right)$ tends to 0 . The reason is that positive customers arrive in the system slowly so that they enter into the limited queue II firstly. With the increase of $p$, queue II is full of customers and the new arrivals get into queue $\mathrm{I}$, which lead to the increasing of $E\left(L_{1}\right)$. When $p$ is constant $(p>0.3)$, we observe that the average length for the RCE killing strategy is shorter than the corresponding for the RCH killing strategy and the value of $p$ is greater; the difference between two killing strategies is also greater.

Figure 3 presents the behavior of $E(W)$ with the parameter $p$. As to be expected, $E(W)$ increases with the increasing of $p$ and the growth rate grows faster and faster. If $p$ is a constant value, the average waiting time for the $\mathrm{RCH}$ killing strategy is greater than the corresponding for the RCE killing strategy. The difference between two killing strategies increases as $p$ increases.

Figures 4 and 5 are drawn in the condition of $\mu_{1}=0.1$, $\mu_{2}=0.3, p=0.4$, and $K=10$. The effect of parameter $q$ on $E\left(L_{2}\right)$ can be found in Figure 4 . Since the arrival of the negative customers can be thought as the speeding up 


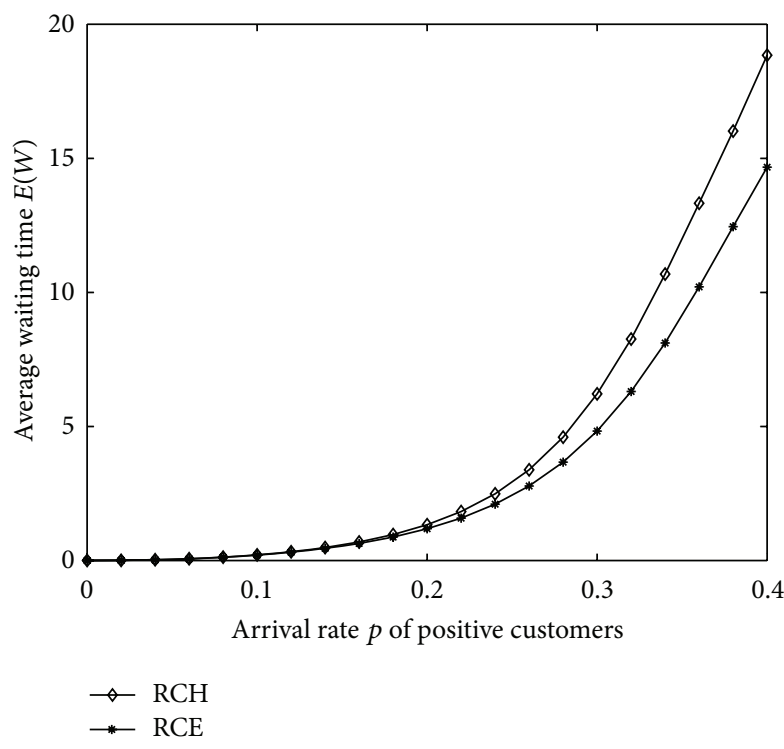

Figure 3: The effect of $P$ on $E(W)$.

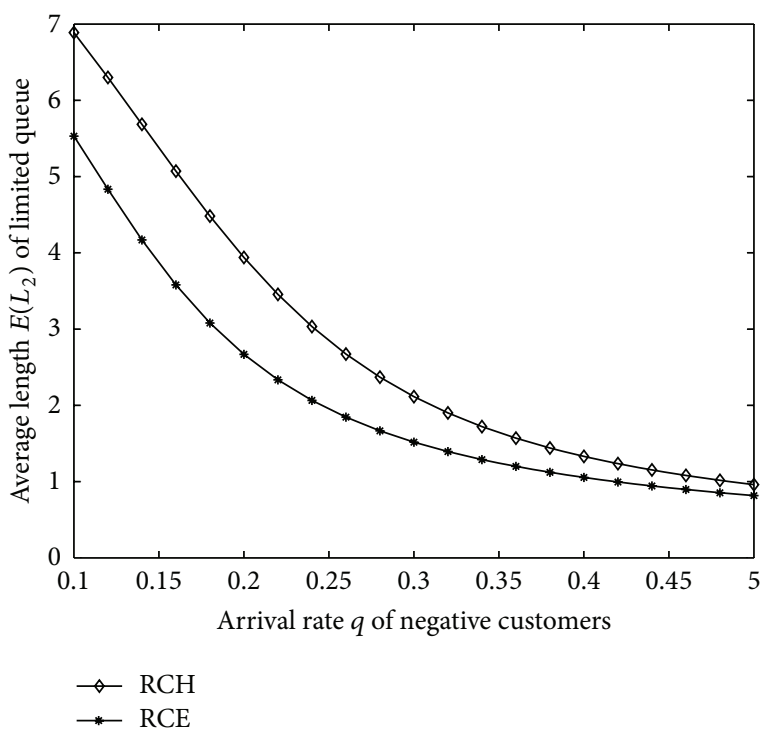

FIgURE 4: The effect of $q$ on $E\left(L_{2}\right)$.

of the service rate, more positive customers leave the system with the increasing of $q$ which leads to the decreasing of $E\left(L_{2}\right)$. In addition, the average length of the limited queue for the RCH killing strategy is greater than the corresponding for the RCE killing strategy, and the difference between two killing strategies decreases with $q$.

Figure 5 shows the relationship between the probability of servers in busy period $P_{b b}$ and the arrival rate $q$ of negative customers. It can be seen that $P_{b b}$ decreases as $q$ increases. With the continuous decreasing of $q, P_{b b}$ tends to 0 . The reason for this is that the arrival rate of negative customers is greater than the arrival rate of positive customers, and negative customers reduce the positive ones until the system become empty.

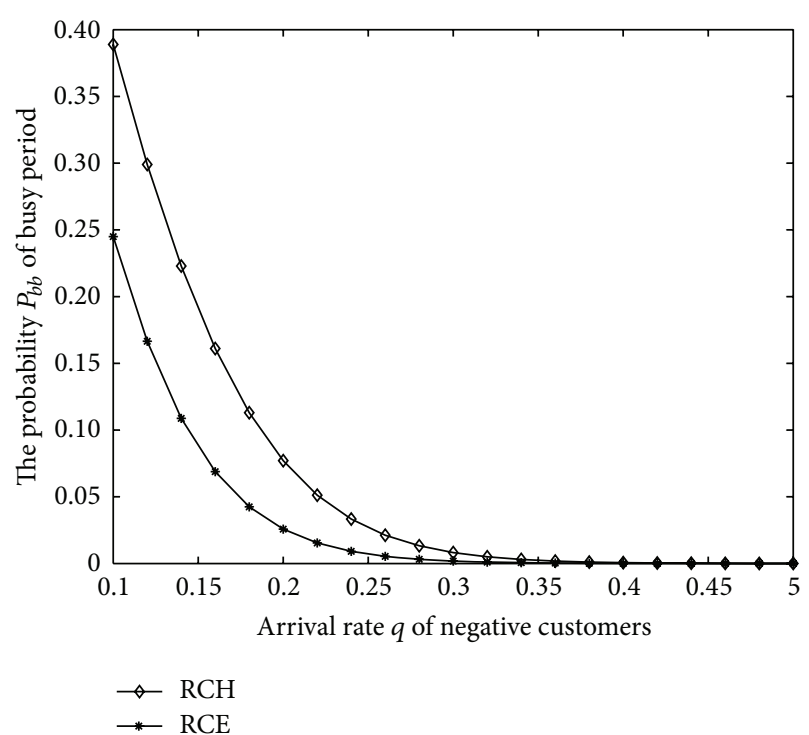

Figure 5: The effect of $q$ on $P_{b b}$.

\section{Conclusion}

In this paper, we applied the RCH and RCE killing strategies to analyze a Geo/Geo/1+1 queueing system. By the method of Quasi-Birth-Death process and matrix geometric solution, we obtained the stationary distribution of the queue length of the considered system. Further, the expressions for some performance measures were derived such as the average waiting time and the probabilities of severs states. We provided the numerical examples to investigate the reason for the influence of some parameters on the system measures.

\section{Acknowledgments}

This work was supported in part by the National Natural Science Foundation of China (no. 71071134), Hebei Province National Science Foundation (no. A2012203124, F2012203093, G2013203169), and Scientific Research Project of Education Department of Hebei Province (no. Z2010182), China.

\section{References}

[1] E. Gelenbe, P. Glynn, and K. Sigman, "Queues with negative arrivals," Journal of Applied Probability, vol. 28, no. 1, pp. 245$250,1991$.

[2] G. Jain and K. Sigman, "A Pollaczek-Khintchine formula for M/G/1 queues with disasters," Journal of Applied Probability, vol. 33, no. 4, pp. 1191-1200, 1996.

[3] R. J. Boucherie and O. J. Boxma, "The workload in the M/G/1 queue with work removal," Probability in the Engineering and Informational Sciences, vol. 10, no. 2, pp. 261-277, 1996.

[4] P. G. Harrison and E. Pitel, "The M/G/1 queue with negative customers," Advances in Applied Probability, vol. 28, no. 2, pp. 540-566, 1996. 
[5] I. Atencia and P. Moreno, "The discrete-time Geo/Geo/1 queue with negative customers and disasters," Computers and Operations Research, vol. 31, no. 9, pp. 1537-1548, 2004.

[6] J. T. Wang and P. Zhang, "A discrete-time retrial queue with negative customers and unreliable server," Computers and Industrial Engineering, vol. 56, no. 4, pp. 1216-1222, 2009.

[7] S. F. Jia and Y. H. Chen, "The Geom/Geom/1 queue with negative customers and single working vacation," Mathematic Application, vol. 25, pp. 304-310, 2012.

[8] X. L. Su and Z. Y. Ma, "The Geom/Geom/1 queue with negative customers, Bernoulli feedback and vacation," Mathematics in Practice and Theory, vol. 41, no. 8, pp. 125-129, 2011 (Chinese).

[9] K. C. Chae, H. M. Park, and W. S. Yang, "A GI/Geo/1 queue with negative and positive customers," Applied Mathematical Modelling, vol. 34, no. 6, pp. 1662-1671, 2010.

[10] J. T. Wang, Y. B. Huang, and Z. M. Dai, "A discrete-time on-off source queueing system with negative customers," Computers and Industrial Engineering, vol. 61, no. 4, pp. 1226-1232, 2011.

[11] J. T. Wang, Y. B. Huang, and T. V. Do, "A single-server discretetime queue with correlated positive and negative customer arrivals," Applied Mathematical Modelling, vol. 37, no. 9, pp. 6212-6224, 2013.

[12] N. S. Tian, X. L. Xu, and Z. Y. Ma, Discrete Time Queueing Theory, Science Press, Beijing, China, 2008. 


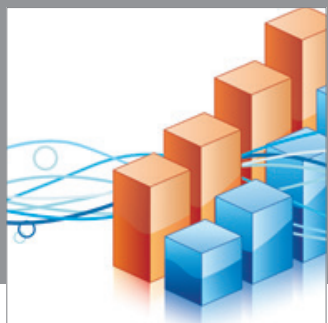

Advances in

Operations Research

mansans

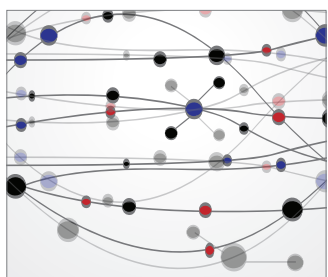

The Scientific World Journal
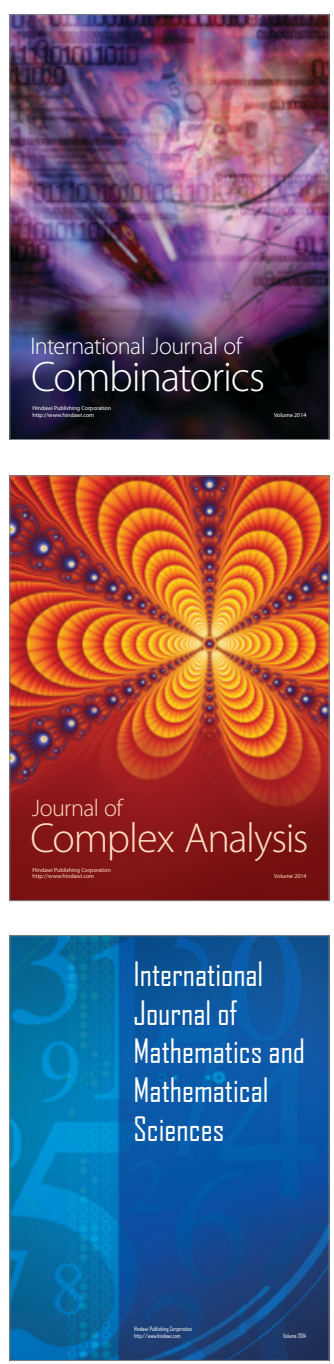
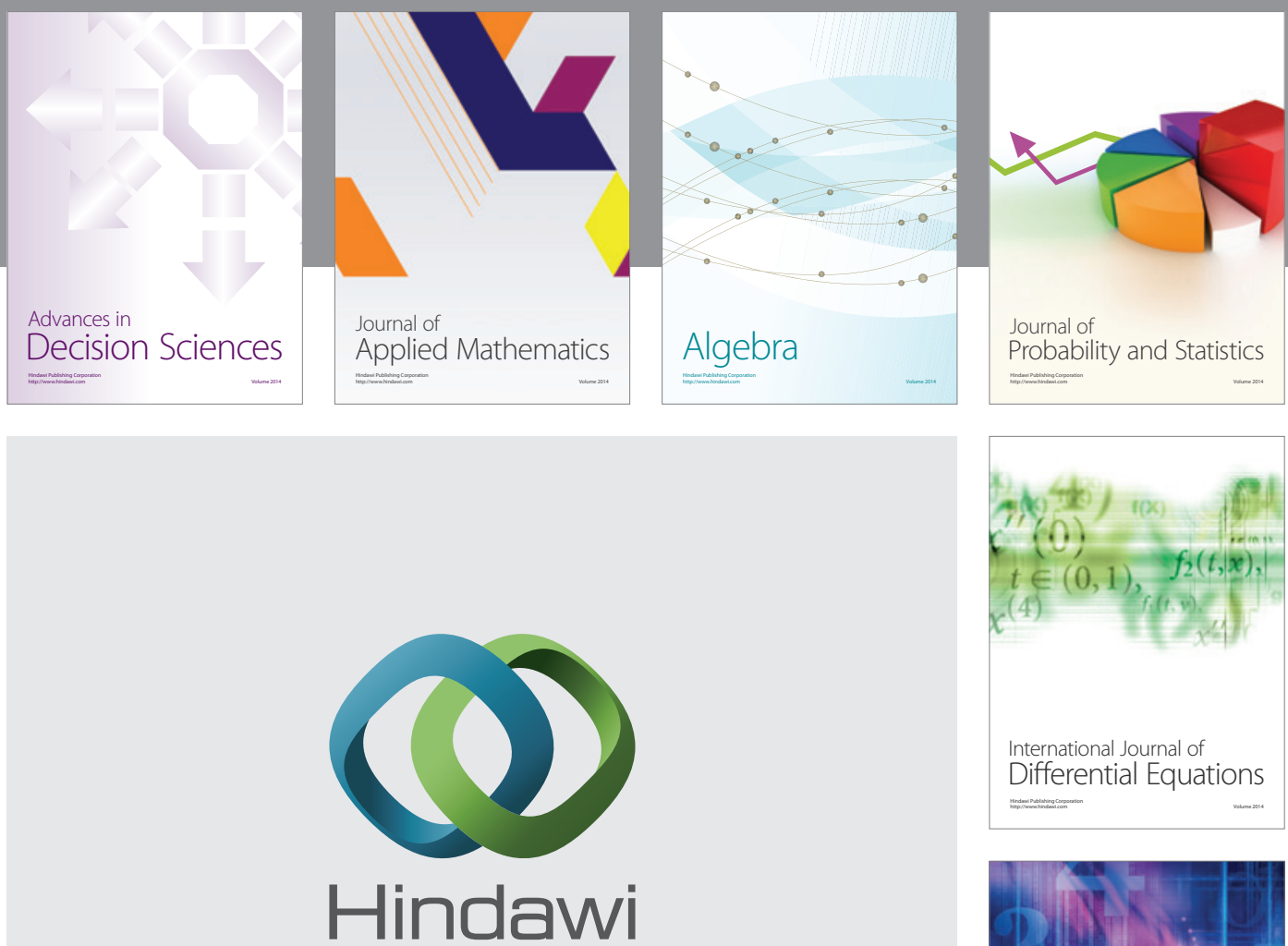

Submit your manuscripts at http://www.hindawi.com
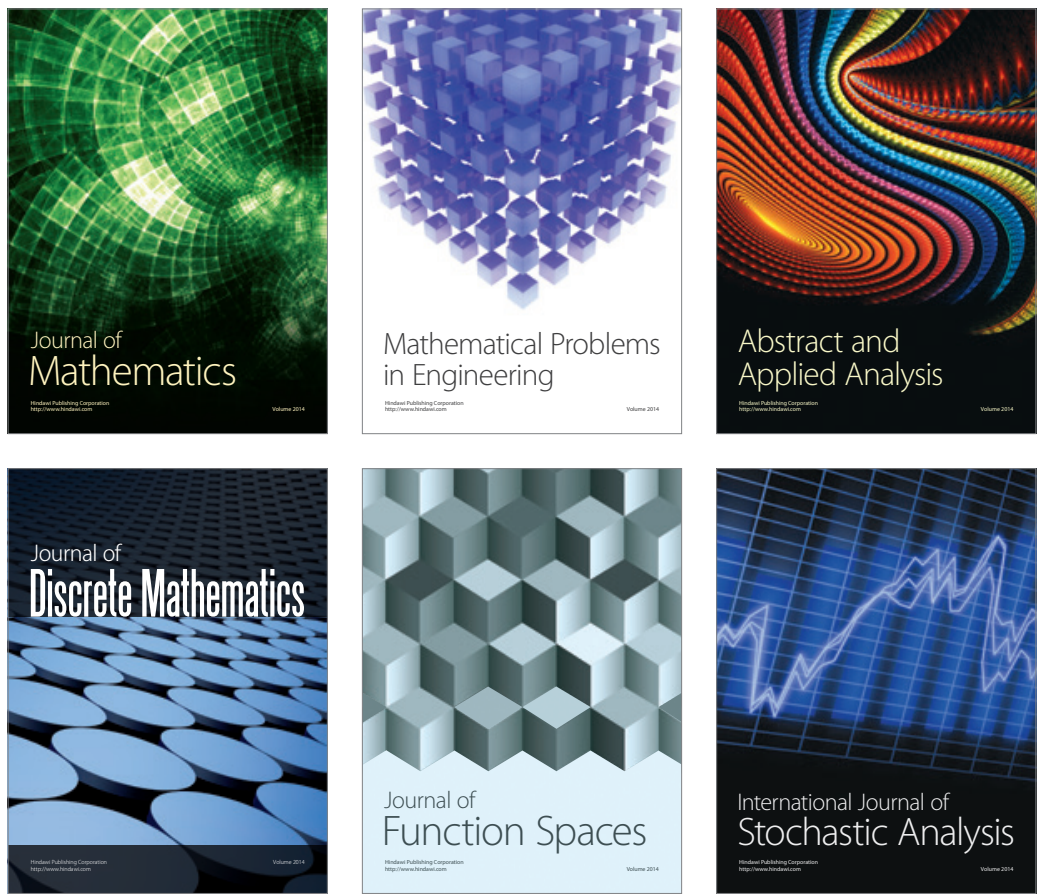

Journal of

Function Spaces

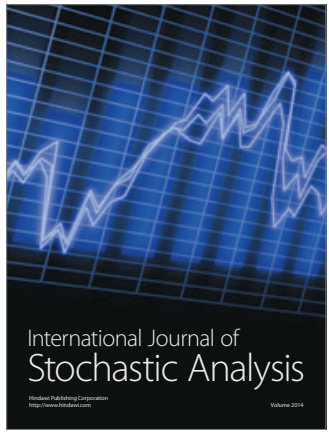

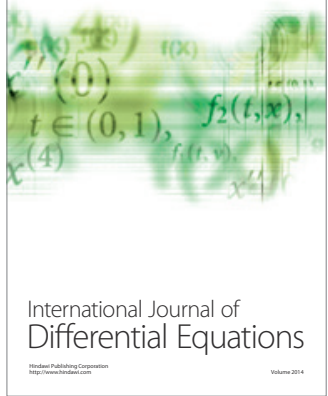
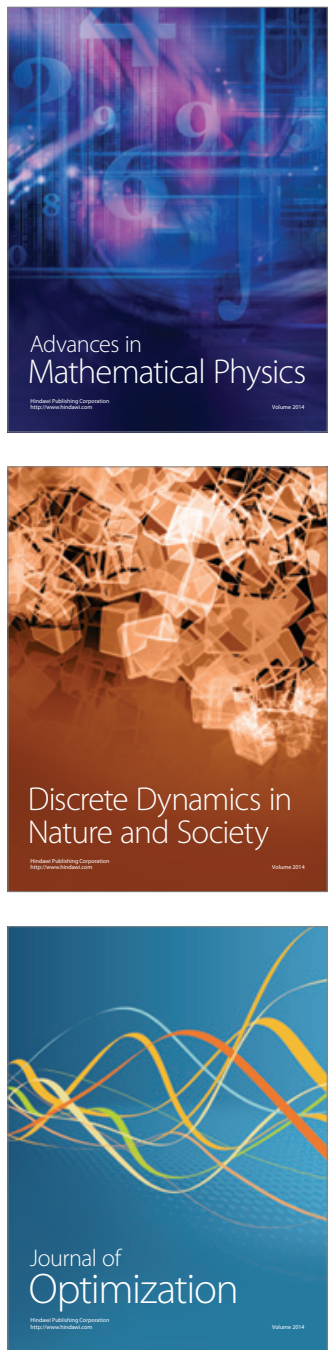